\title{
CSPA: Channel Selection and Parameter Adaptation scheme based on genetic algorithm for cognitive radio Ad Hoc networks
}

\author{
Saleem Aslam and Kyung Geun Lee*
}

\begin{abstract}
The cognitive radio (CR) is evolved as the potential technology to solve the problem of spectrum scarcity and to meet the stringent requirements of upcoming wireless services. CR has two distinct features, the spectrum sensing and the parameter adaptation. The former feature helps the $C R$ to find the vacant spectrum slots/channels in the radio band while the latter mechanism allows it to adjust the operating parameters (e.g. frequency band, modulation and power, etc.) accordingly. The primary user (PU) activity has serious effects on the overall performance of the cognitive radio network (CRN). The CR should vacate the channel if it detects the arrival of the primary user (PU) in order to avoid the interference. The channel eviction/switching phenomenon severely degrade the quality of service (QoS) of the CR user and it is perhaps the key challenge for the CRN. In this paper, we propose the dynamic channel selection and parameter adaptation (CSPA) scheme based on the genetic algorithm to provide better QoS for the CR by selecting a best channel in terms of the quality, the power and the PU activity. The CSPA deals with the problem of channel switchings and it provides better QoS to the CR user. Simulation results prove that CSPA outperforms the existing schemes in terms of channel switchings, average service time, power and throughput.
\end{abstract}

Keywords: Cognitive radio, Genetic algorithm, QoS, Channel characteristics, PU activity

\section{Introduction}

The radio spectrum is the most precious resource in the wireless communication. Each wireless transmitterreceiver pair performs communication by occupying a specific portion of the radio spectrum. According to the latest research figures, the usage of the allocated spectrum band is very limited and it is around $15 \%$ to $25 \%[1,2]$. On the other side of the picture, the current allocation state of the spectrum is overcrowded and there is a small portion of spectrum left available for new emerging wireless services. These factors incline the researchers to devise new ways for the efficient utilization of the existing spectrum. The most feasible and promising solution is the CRN.

The $\mathrm{CR}$ is an intelligent adaptive and opportunistic radio, which can increase the spectrum efficiency by dynamic selection of the unused portion in the radio band

\footnotetext{
* Correspondence: kglee@sejong.ac.kr

Department of Information and Communication Engineering, Sejong University, Seoul, Republic of Korea
}

using the cognitive sensor (CS) and quick reconfiguration of the transmission-reception parameters using software defined radio (SDR). The CR utilizes the idle channels during the available time depending upon the information collected through spectrum scanning or spectrum sensing. In the current modeling of CRN, if the PU arrives on a the channel, then the CR immediately shifts to another channel or remains silent to avoid the interference with the PU. The overall performance of the CRN directly depends upon the spectrum sensing, the PU activity and the variations in the quality of the channel during course of transmission.

Wireless ad-hoc network consists of self-managing radios that operate in a distributive pattern without the need of a central base station. The cognitive radio adhoc networks (CRAHNs) [3] allows the CRs to form a dynamic network without using a CR base station and a fixed spectrum as shown in Figure 1 (b). The CRAHNs are considered to be important because of their key applications in many practical areas including the 


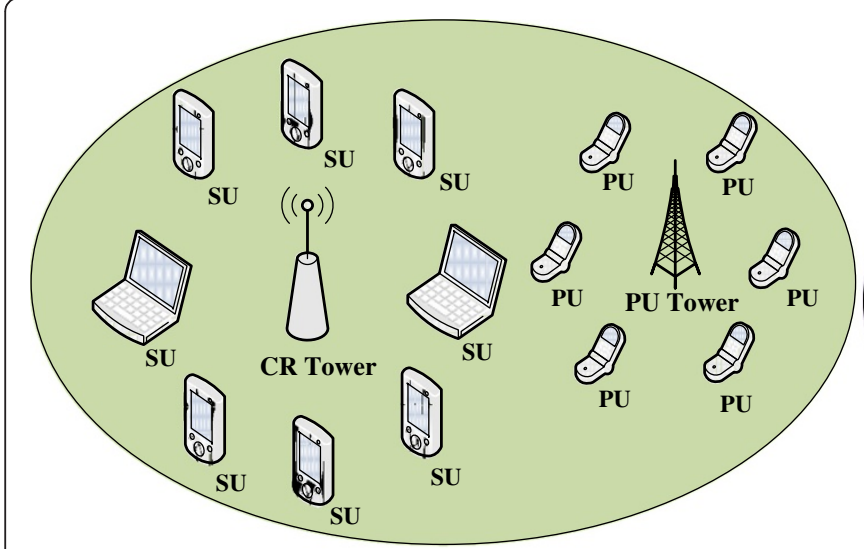

(a)

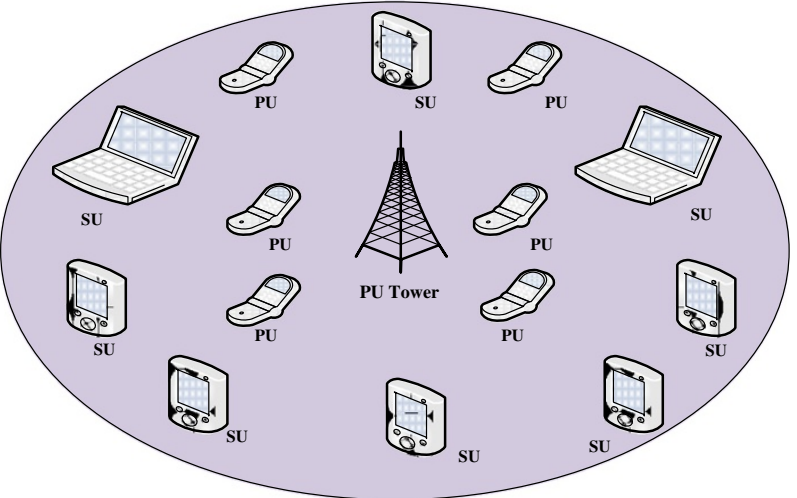

(b)

Figure 1 CRN architecture (a) Infrastructure (b) CRAHN.

communication in the disaster, the battle and the autonomous vehicles [3].

The channel selection is an important area in the CRAHNs. It enables the CR to choose the best channel among the pool of sensed channels. The current research in the area of channel selection relies on the latest observations of the channel state as a base knowledge for the selection mechanism. However, a channel that is to be utilized by the PU cannot be used for the transmission of the CR. Therefore, when the PU arrives, the CR should suspend its communication, and it should look for other channel to restart its communication [4-6]. Such periodic suspensions in the transmission of the CR user can cause severe degradation in their QoS. To deal with such disruptive scenarios, a new model is presented in [7-11]. In these schemes, each $C R$ predicts the future arrival of the PU on different channels and selects the best channels for its communication depending upon the least PU activity. The purpose of these schemes is to minimize the interference with the PUs. However, other factors are also important for the selection of the optimal channel such as the channel condition and the QoS requirement of the CR. A robust channel selection scheme should accommodate all these factors for the performance evaluation of the CRN. Moreover, these factors are contradictory in nature and demand an intelligent and an effective solution.

The genetic algorithm (GA) seems to be the best solution owing to its multi-objective optimization capability [12]. It can be employed to solve the problems which have large search space and contradictory objectives. In the latest research models $[13,14]$, the authors describe the cognitive decision making process (DMP) using the GA to solve the multi-objective optimization problem in the CRN. However, their schemes only consider the channel condition as the prime factor and lacks in terms of the PU activity and CR QoS requirements.

Similar GA based schemes presented in [15-19] perform the channel selection by considering the power (PWR), modulation (MOD), bit error rate (BER), bandwidth (B) and frequency as the basic genes. In these schemes, the objective function converges to the optimal value and then termination condition is achieved based on the desired criteria. Although theses schemes provide the detailed analysis and implementation of the channel selection scheme to optimize the factors such as data rate (DR), PWR and BER but these schemes also don't consider the PU activity and CR QoS parameters.

Another GA based parameter adaptation scheme is presented in [20]. It covers the detailed description of various GA parameters and provides a comprehensive simulation model for the parameter reconfiguration. The authors select the three basic objective functions such as minimize BER, minimize power and maximize throughput and provide the simulation results for two different objective function's weight scenarios termed as emergency and low power. Although simulation results show better comparisons in terms of throughput, BER and power like [16-18], yet all of these schemes lack in accommodating the effects of the PU activity on the performance of the CRN.

In this paper, we propose a GA based channel selection and parameter adaptation (CSPA) scheme by considering the PU activity, the QoS of secondary user (SU) and the channel condition and quality under one umbrella. According to best of our knowledge the PU activity has not been considered in the context of GA based channel selection scheme. The CSPA scheme incorporates the effects of the PU activity by predicting the future opportunity to $\mathrm{CR}$ user the sensed channels. We add this information as a new gene in the chromosome 
structure of the CSPA scheme and show the effectiveness of our scheme in terms of channel switchings, average service time, power and throughput using Matlab simulations. The main contributions of this paper can be summarized as follows:

i) We formulate the problem of channel selection and parameter adaptation in CRAHNs using GA by considering all the relevant parameters (BER, PWR, DR, etc.) and other important factors (Channel condition, the PU activity and the CR QoS requirement) from the perspective of both the $\mathrm{PU}$ and the CR. We provide an analysis of existing GA based schemes and discuss the challenges and issues involved in the design of such schemes. This is a valuable contribution for future research in this direction.

ii) We propose a method to model the arrival activity of the PU and also formulate the mathematical equation to compute the transmission opportunity index (TOI). The TOI represents the future channel availability to the $\mathrm{CR}$ without interruptions from the PU.

iii) Finally, we compare our proposed scheme with the existing random channel selection schemes in terms of the channel switchings, the service time, throughput and the power consumption.

The rest of paper is organized as follows. Section Problem formulation describes the problem formulation of the CSPA scheme. The GA, its applications and its modeling with respect CRAHNs is presented in Section Genetic algorithm. Section Experimental results explains the experimental setup and simulation results of our proposed scheme. Finally, Section Conclusion draws the conclusion and sets the future research direction for the design of the channel selection scheme in CRAHNs.

\section{Problem formulation}

We consider a network with $m=1,2,3 \ldots M$ PUs and $n=1,2,3 \ldots N$ CR users operating in a similar model as shown in the Figure 1 (b). The CR users operate in an ad hoc or distributive fashion with the prime objectives to maximize the overall spectrum usage, to minimize the channel switchings and to reduce the energy/power consumption. The entire operation of our CSPA system can be described as follows:

i) Each CR user $n=1,2,3 \ldots N$ periodically senses the radio environment to gather the statistics about the PUs which are operating in the vicinity under the PU base station as shown in Figure 2.

ii) For spectrum sensing, the CR user employs the cyclo-stationary feature detection (CSFD) scheme as shown in Figure 2. We prefer CSFD sensing scheme for its two basic advantages as compared to the other transmitter based spectrum sensing schemes. Firstly, it can detect the presence of the PU transmitter reliably. Secondly, it can discriminate the waveform of different wireless technologies. In our case, reliable detection (low probability of false alarm and miss detection) is extremely important because each $\mathrm{CR}$ user needs to maintain the history of channel based on its sensing results. And, also discrimination property is desirable in our scheme because we are considering the $\mathrm{CR}$ network operating with multiple PU networks. The CSFD can easily differentiate between the PU signals of different technologies. For example, it can easily classify the signal of the CDMA and the GSM

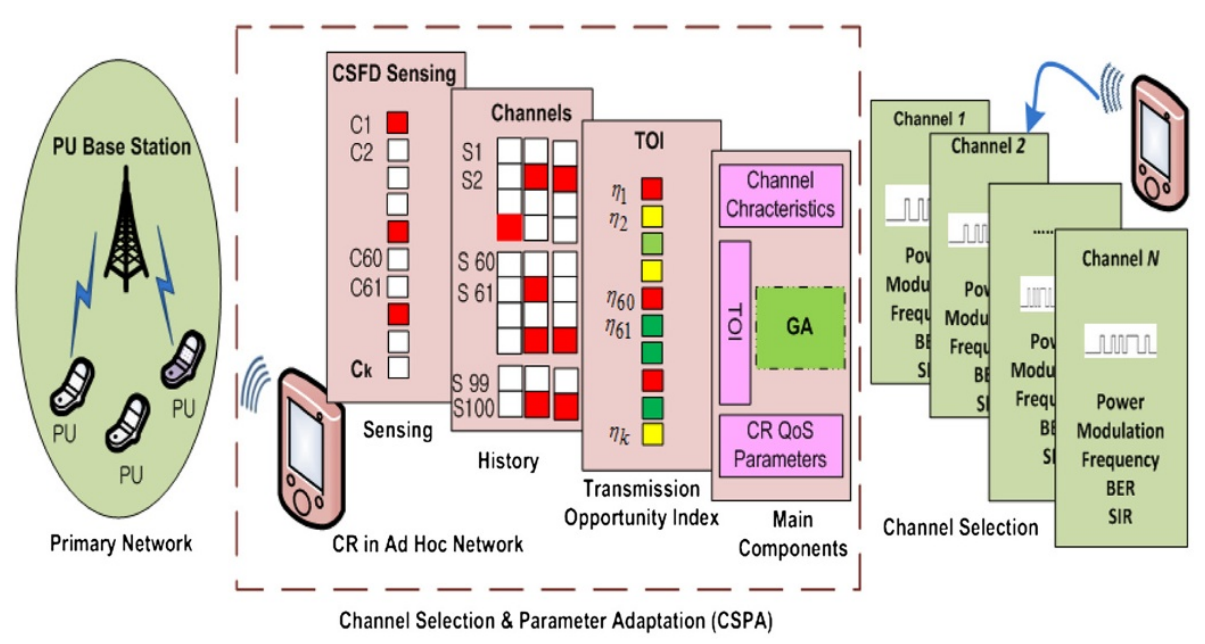

Figure 2 System model. 
technology. This discrimination property helps the CR to quickly adapt its parameters according to the desired technology. The entire working of CSFD can be represented by the following equations presented in [21-23] and depicted in Figure 3. The threshold value of $\lambda$ indicates about the presence or absence of the PU. The spectral contents of the waveform of the PU's signal over the time interval $[\mathrm{t}-\mathrm{T} / 2, \mathrm{t}+\mathrm{T} / 2]$ can be computed by the relationship given in [21] as follows

$$
P_{T}(t, v)=\int_{t-T / 2}^{t+T / 2} p(u) e^{-j 2 \pi v u} d u
$$

where, $v$ represents the evaluated frequency and it represents the spectral components with separation factor $\alpha$.

The spectral correlation function can be represented by the two spectral components of the PU signal $\mathrm{p}$ (t) at the frequencies $v=f \pm \alpha / 2$ can be represented using equation (2).

$$
\begin{aligned}
S_{p T}^{\alpha}(f)_{\Delta t} & =\frac{1}{\Delta t} \int_{-\Delta t / 2}^{\Delta t / 2} \frac{1}{\sqrt{T}} P_{T}(t, f+\alpha / 2) \cdot \frac{1}{\sqrt{T}} \\
& \times P_{T}^{*}(t, f-\alpha / 2) d t
\end{aligned}
$$

The spectral components of the PU signal can be measured in ideal condition using the equation (3).

$$
S_{p}^{\alpha}(f)=\lim _{T \rightarrow \infty \Delta t \rightarrow \infty} \lim _{p T} S_{\Delta t}^{\alpha}(f)_{\Delta t}
$$

The decision about the cyclic frequency is taken depending upon the value of the coherence function depicted in the [20] and given in equation (4).

$$
C_{p}^{\alpha i}(f)=\frac{S_{p}^{\alpha i}(f)}{\left[S_{p}(f+\alpha i / 2) S_{p}(f-\alpha i / 2)\right]^{1 / 2}}
$$

If the coherence function is greater than the certain threshold then it declares that the $\alpha_{i}$ is the cyclic frequency. After checking all possible values of $\alpha_{i}$, the CSFD collects the cyclic frequency and compare it with the waveform available in the data base and then provides indication about the presence or absence of the PU on the particular frequency $f$.

iii) Each CR user maintains the channel history from the sensing results obtained from the CSFD scheme.

$$
\Phi_{s}^{k}=\left[\Phi_{s}^{1}, \Phi_{s}^{2}, \Phi_{s}^{3}, \ldots, \Phi_{s}^{k}\right]
$$

where, $\Phi_{s}^{k}$ represent the PU activity vector during time slot $s$ and $k$ indicates the particular channel.

$$
\Psi_{s}^{k}=\left[\Psi_{1}^{k}, \Psi_{2}^{k}, \Psi_{3}^{k}, \ldots, \Psi_{s}^{k}\right]
$$

where $\Psi_{s}^{k}$, indicates the history vector of a particular channel $k$ for $s$ number of time slots. The value of $s$ for our case is 100 as shown in Figure 2.

$$
\mathrm{H}=\left\{\begin{array}{ccccc}
\Psi_{1}^{1} & \Psi_{2}^{1} & \Psi_{3}^{1} & \ldots & \Psi_{s}^{1} \\
\Psi_{1}^{2} & \Psi_{2}^{2} & \Psi_{3}^{2} & \ldots & \Psi_{s}^{2} \\
\Psi_{1}^{3} & \Psi_{2}^{3} & \Psi_{3}^{3} & \ldots & \Psi_{s}^{3} \\
\vdots & \vdots & \vdots & \vdots & \vdots \\
\Psi_{1}^{k} & \Psi_{2}^{k} & \Psi_{3}^{k} & \ldots & \Psi_{3}^{k}
\end{array}\right\}
$$

The $H$ matrix forms the history patterns for the PUs on the $k$ number of channels. The size of history matrix is $k * s$. The increase in the size increases the reliability but the system becomes more complex. We select the normal values for both $k$ and $s$ to show the outcomes and advantages achieved through CSPA.

The history of each channel is maintained and then based upon the history we calculate the transmission opportunity index (TOI) for each available channel as indicated in Figure 2. The TOI quantifies the future availability of channel for the

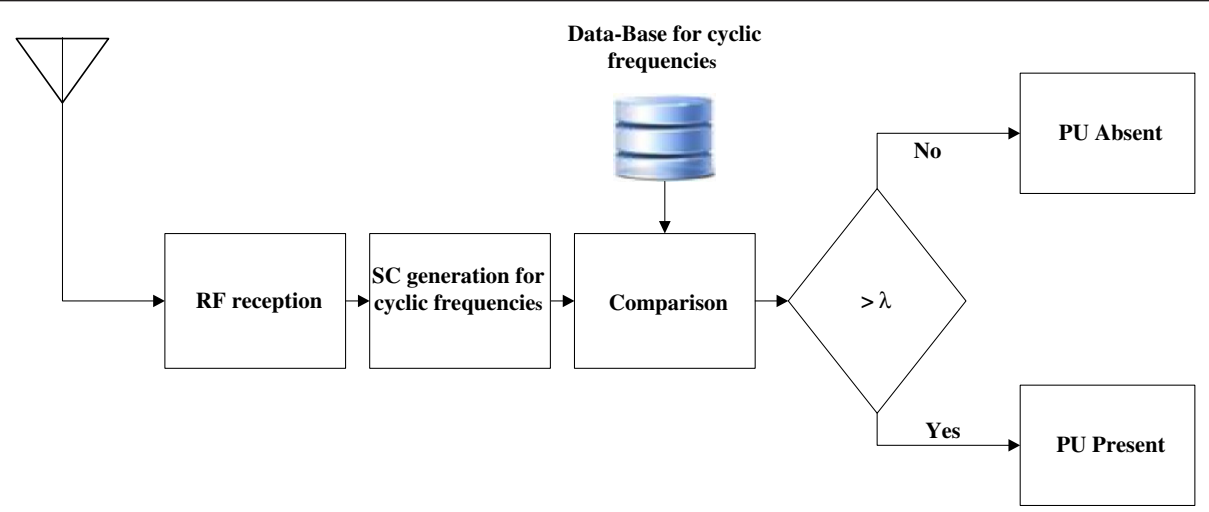

Figure 3 Block level representation for cyclostationary feature detection. 
CR communication without PU interruptions. The TOI can be calculated by the procedure depicted in Figure 4. Mathematically, TOI can be represented by the following equation (8)

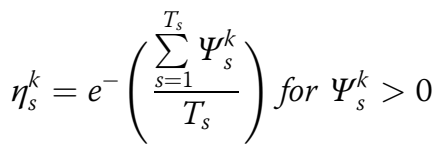

Where $\eta$ represents TOI and $\Psi$ indicates the history pattern of the PU on the channel for Ts number of time slots and it is calculated using equation (5). The value of summation varies from 0 to 1 while the TOI $(\eta)$ varies from 0.3697 to 1 . The variables $\mathrm{Ts}$ and $s$ can be used interchangeably and these are used together to improve clarity.

The TOI can help to achieve the following main objectives:

-Minimization of switching overhead.

- Minimization of the interference.

- Minimization of energy by reducing channel switchings.

- Better QoS to CR user.

iv) The CSPA selects the best channel in terms of the CR QoS requirement, the condition of channel and the activity of the PU. The CRN can be characterized with three basic entities i.e. the PU, the $C R$ and the channel. Each entity has unique features and the nature of these features strongly affect the overall performance of the CRN. The CR user has unique requirements from the channel depending upon its QoS parameters. However, the PU has prime concern from CR that it should operate under tolerable interference limit. The
Figure 5 depicts the main entities involve in the channel selection of CRN.

\section{Genetic algorithm}

This section covers the background and detailed description of the genetic algorithm. Moreover, we also provide the gene and chromosome structure along with the explanation of the objective functions of CSPA algorithm in this section.

\section{Application and background}

The GA is a biologically inspired heuristic search technique based on the phenomenon of natural genetics. The GA maintains the population of individuals that characterize the candidate solutions to the given problem. Each individual chromosome in the population is evaluated to find its fitness level (how much it is close to the optimal solution) from the given objective functions. The chromosomes consist of different genes and these genes can be represented through the binary numbers $[0,1]$.The complexity of chromosome is directly depends upon the number of genes and the bits/gene.

The GA performs well for large search space problems because it can work on a population in parallel instead of processing a single solution at a time. The parallel processing allows the GAs to explore several parts of the solution at same time [6] and also many real world problems require simultaneous optimization of several objective functions. Hence, these algorithms become suitable to solve these problems. However, the objective functions may have conflict in their objectives [22]. These contradictory requirements of different objective functions give rise to a set of possible solutions call pareto optimal solutions instead of a single possible solution. The main reason for the multiple possible solutions
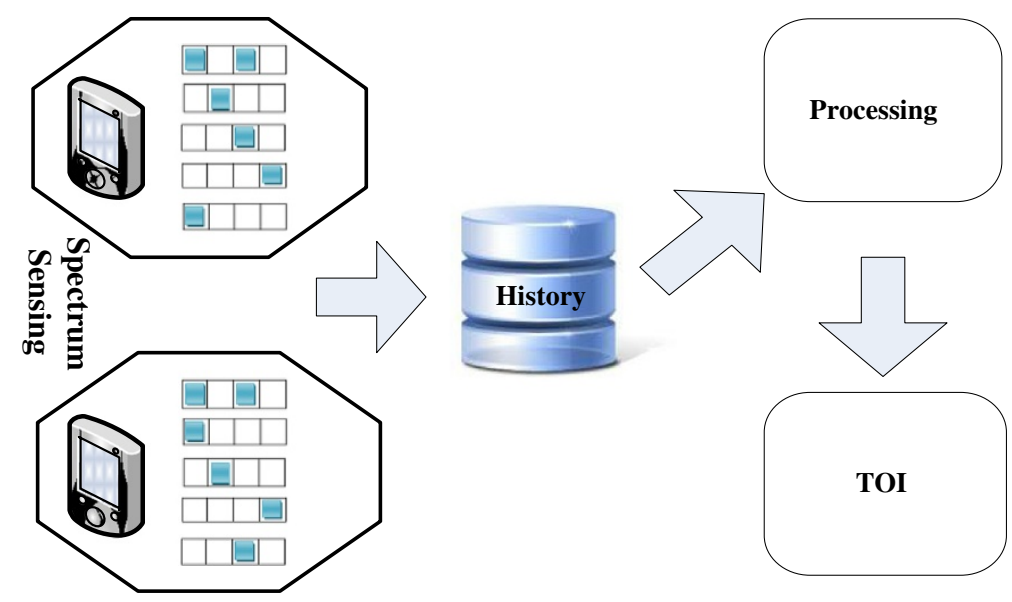

Figure 4 Transmission opportunity index (TOI). 


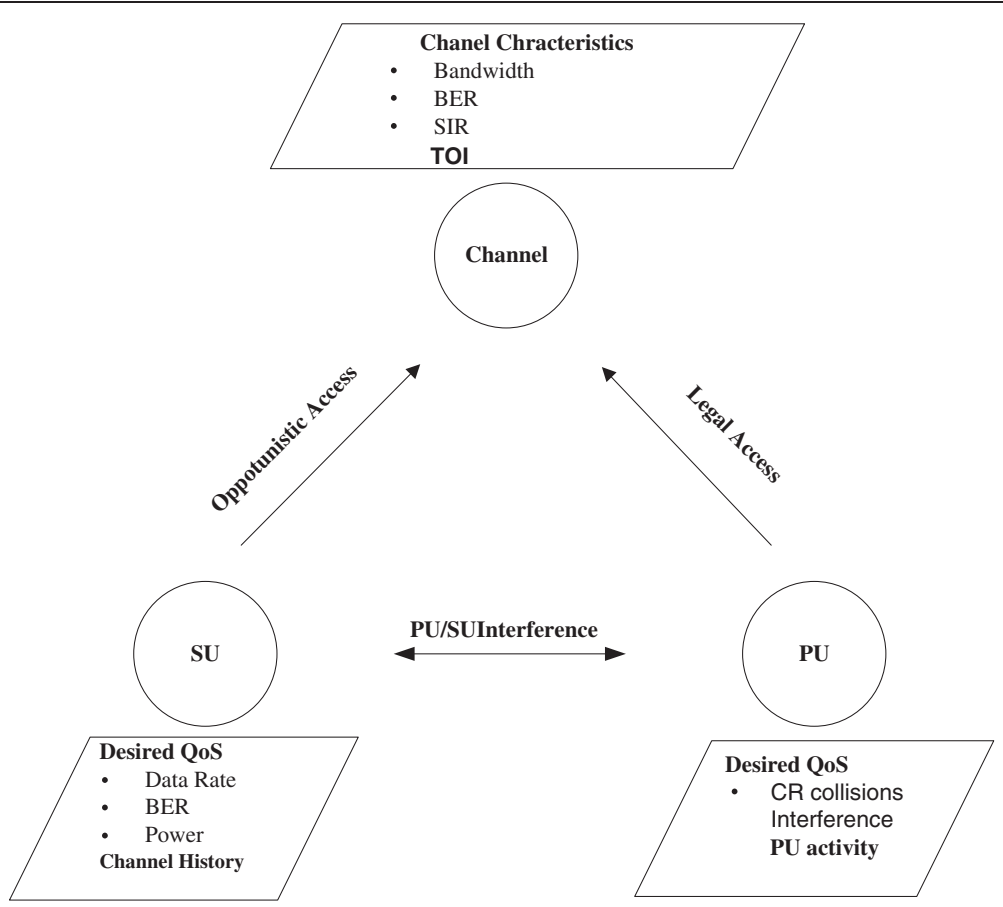

Figure 5 The prime factors for channel selection in CRN.

is that no single solution can be thought as the best one than the other solution in the optimal set. The working of GA can be described by the algorithm 1 .

\section{Algorithm 1: Basic steps in the GAs}

1. Initialize the population of chromosomes.

2. Compute the fitness level of each chromosome to rank them.

3. Select the best chromosomes in terms of their fitness.

4. Perform the crossover operation on selected chromosomes.

5. Perform the mutation function on selected chromosomes.

6. If stopping criteria is achieved terminate the GA otherwise move to step 2 of the algorithm.

The brief explanation of each step involved in the GA is as follow.
A. Initialization
An initial population with $i$ random chromosomes is generated. These chromosomes contain the available solutions to the given problem under analysis.
B. Fitness Evaluation(FE)
$\mathrm{FE}$ represents the evaluation of fitness of random generated chromosomes.
C. Formation of New Generation

This component performs the following steps to reproduce the chromosomes until the next generation completes.

i) Selection: The chromosomes are selected in such a way to have the better level of fitness in the current available population.

ii) Crossover: The crossover is an operator to share information between chromosomes and to create new individuals from the incoming generation.

iii) Mutation: The new created individual will be mutated at a definite point in the chromosome.

\section{Implementation of the GA in CRN}

The channel selection and parameter adaptation is the important part in the implementation of the CRN. At one end, the CR should select the best channel in terms of the factors mentioned in Figure 5 while on the other end it needs to quickly adjust its operation parameters according to the selected channel. We focus on the channel selection and parameter adaptation for CRAHNs with a hypothesis that inputs are gathered through spectrum sensing, QoS of the CR and condition of channel. In order to provide the better QoS in terms of data rate, service time (delay) and minimal interruptions (channel switching), we add a new TOI gene inside the chromosome. The TOI gene helps to save transmission power by reducing the number of retransmissions that occurs because of the collisions with the PU. 
Figure 6 explains the entire working of CSPA algorithm based on the GA. We explicitly show the channel history and TOI to clarify our contribution and to show how we incorporate the PU activity.

\section{Encoding of chromosomes}

The genes are the basic building blocks of the GAs. The combination of genes forms the chromosome. The chromosome depicts the entire information about the solution of the given problem. In current article, we design the GA for an individual CR operating in an ad hoc fashion. There are seven genes including frequency band (FB), modulation (MOD), bit error rate (BER), power (PWR),

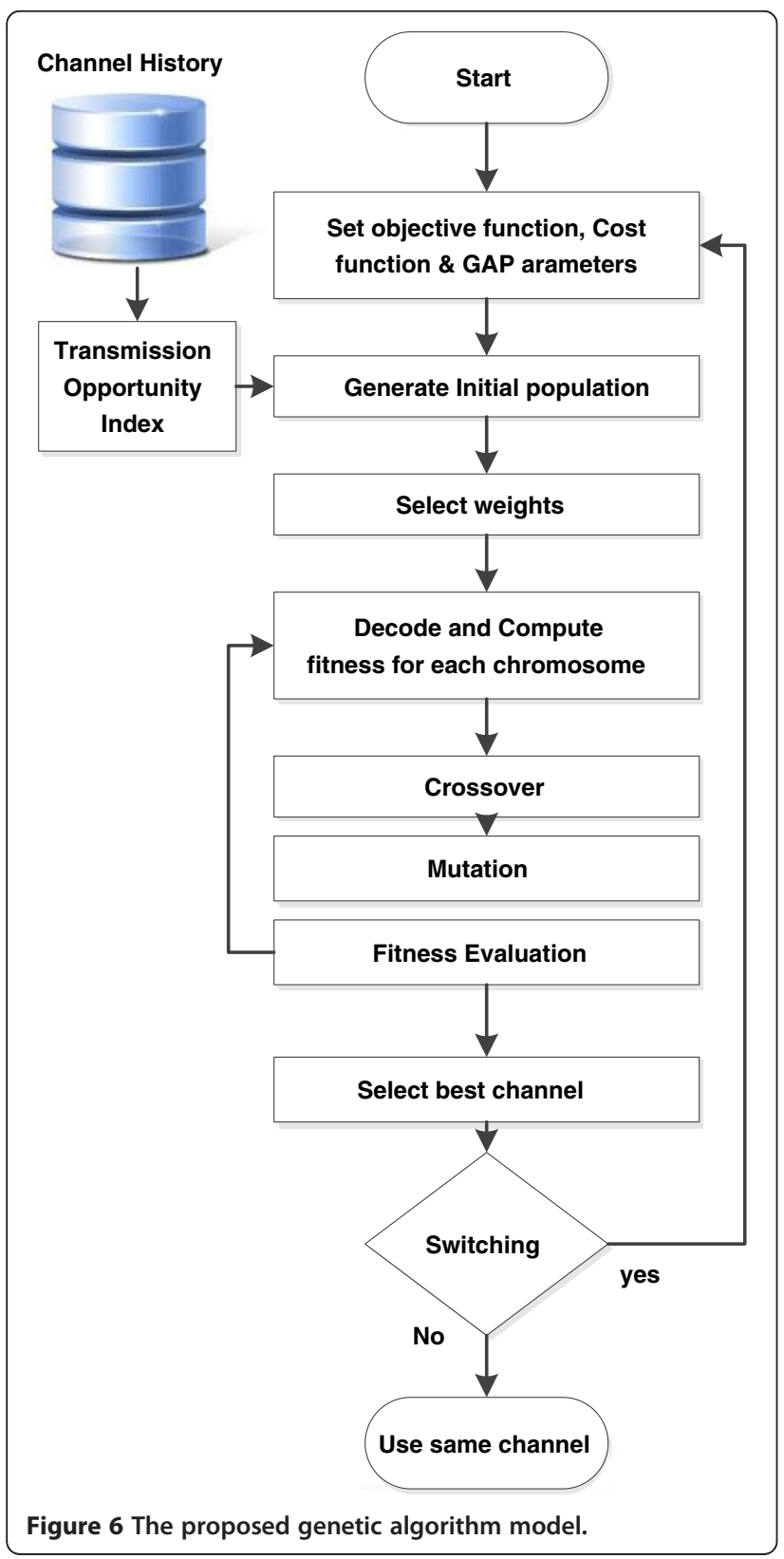

data rate (DR) interference to primary user (ITPU) and TOI. The combination of these seven genes forms a complete 30 bit solution (chromosome) and the encoding of the genes can be performed as follows.

i) Frequency band (FB)

The FB is the first gene in our proposed CSPA scheme used for the generation of chromosomes. In wireless communication, each transmitter requires a spectrum band for its operation. In current communication environment, each technology is assigned with fixed spectrum band while the CR can operate on any available band. In current scheme, we use the same spectrum band specifications that are given in IEEE 802.22. The $\mathrm{FB}$ varies from $54 \mathrm{MHz}$ to $862 \mathrm{MHz}$ with step size of $8 \mathrm{MHz}$. Therefore, FB gene requires 7 bits for encoding process. The encoding variable varies from 0000000 to 01100011 . The explanation of the FB gene is described in Additional file 1: Table S1.

ii) Modulation (MOD)

The MOD is the second gene in our scheme. In wireless communication, Mod states the relationship between two different types of waveforms. In current scheme we use the same specification for the MOD that is given in IEEE 802.22. The four modulation schemes are BPSK, QPSK 8-QAM and 16-QAM and the encoding variable is varying from 00 to 11 .

iii) Bit error rate (BER)

The next important parameter or gene for wireless communication is the BER. It defines the number of received bits that have been altered due to noisy channel or interference. Each application can tolerate BER up to the certain limit. For example, video streaming can tolerate the higher bit error rates while voice applications are very sensitive to BER. Moreover, BER is extremely important for wireless media owing to the more external noise and interference. In our work, BER ranges from 10${ }^{1}$ to $10^{-16}$ as mentioned in most of existing schemes $[19,20]$ and the step size is $10^{-1}$ and hence it requires 16 levels and four bit for the gene representation. The detailed decimal representation of the BER gene is shown in Additional file 1: Table S1.

iv) Data rate (DR) The next important gene is the DR. It defines the number of bits transmitted in a unit time. It can be represented using bits per second (bps) or kilobits per second (kbps). Each application requires a reasonable DR and it varies across the applications. For example, video streaming requires higher data 
rate while voice applications require comparatively less data rate. The DR varies from $50 \mathrm{kbps}$ to $1.65 \mathrm{Mbps}$ with step size of 50kbps. There are total 32 possible data rates and hence it requires 5 bits for encoding process. The encoding variable varies from 00000 to 11111. The gene structure for DR is described in Additional file 1: Table S1.

v) Power (PWR)

The fifth important parameter for wireless system is the power. For current article we take the power range from $3 \mathrm{dBm}$ to $48 \mathrm{dBm}$ with step size of $3 \mathrm{dBm}$. We convert the $\mathrm{dBm}$ values to miliwatts $(\mathrm{mw})$ for simplicity and represent the simulation results in mw. This requires 16 levels to represent this gene of the chromosome in decimal format. The entire power band is shown in Additional file 1: Table S1. To get the desired power for an application, the CR user can input a decimal value. For Example, if the application desires $24 \mathrm{dBm}$ of power then the CR user can specify its desired value as binary vector 1000 .

vi) Interference to primary user (ITPU)

Another important parameter for the CRN is the ITPU. It occurs because of the transmission of the $\mathrm{CR}$ on the ongoing communication of the PUs. The transmission power of the CR can degrade the performance of the PUs. For simplicity, we consider the ITPU gene from 0.0625 to 1 with step size of 0.0625 . This requires 16 levels to represent this gene and explanation is given in Additional file 1: Table S1.

vii) Transmission opportunity index (TOI) The most important parameter for channel selection process is the random PU arrival activity. The TOI defines the future opportunity of a channel for the CR. The larger the value of the TOI, the large available time for the CR user and there will be less interruptions from the $\mathrm{PU}$ and hence the CR can get a reasonably good QoS for its communication. If the CR selects the channel without considering the PU activity on random bases then there are more chances of collisions with the PU. We maintained the history for the PU activity on each channel and then select the channel with least PU activity. The least PU activity corresponds to the higher value of TOI. . By selecting the channel based on the PU activity, throughput and minimal power significantly improves the QoS for the CR user. We consider the simplest gene structure for the TOI with 16 possible levels and details are given in Additional file 1: Table S1.

viii) Bit level representation of chromosome The seven genes constitute the structure of chromosome. The 30-bit chromosome represents the complete solution of CSPA scheme using 30 bits. The arrangement of all genes in the chromosome is shown in Figure 7.

\section{Fitness measure}

The next important step after the formation and initialization of the population of the chromosomes is the fitness computation. Fitness measure indicates the capability of chromosome to solve the given problem. The fitness level can be represented by a number ranging from 0 to 1 , where 1 represents the best fitness level. The fitness of a chromosome can be represented using $R_{n}$.

$$
R_{n}=\sum_{i=1}^{n} w_{i} \cdot f_{i}
$$

\section{Selection of chromosome}

In order to form the next generation the selection process sorts out the chromosomes in descending order depending upon the value extracted through equation (9). Suppose there are $N_{c}$ number of chromosomes in the initial stage and $N_{s}$ represents the selected chromosomes and $N_{s r}$ indicates selection rate then the selected chromosomes can be represented as follows.

$$
N_{s}=N_{c} * N_{s r}
$$

The selection rate depends upon the complexity and convergence time and we choose the selection rate equals to $1 / 2$.

\section{Crossover}

The crossover is a process in which characteristics of a pair of parent chromosomes are exchanged with each other to form a pair of child chromosomes. This step is also known as reproduction. There exists several schemes such as single point, 2-point, multipoint and uniform crossovers. In this article 2-point crossover has been implemented. For example, the formation of two child chromosomes $\mathrm{c} 1$ and $\mathrm{c} 2$ from two parent chromosomes is depicted in Figure 8. The Figure 8 (a) indicates the bit level representation for the first parents while the Figure 8 (b) represents the parent 2 at bit level. The formation of the child 1 as a result of crossover between parent 1 and parent 2 is depicted in Figure 8 (c) and 8 (d).

\section{Mutation}

The next step after forming the new generation is the mutation process. Mutation alters a binary bit of 1 to 0 or vice versa in the child chromosomes. 


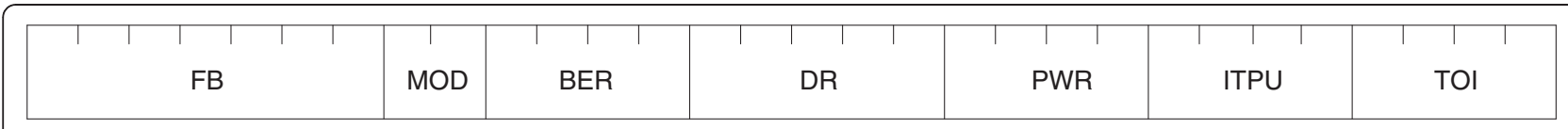

Figure 7 Chromosome structure.

The mutation rate indicates that how many chromosomes out of the total chromosomes undergo the mutation process. For example, the mutation rate of 0.03 indicates that 3 chromosomes out of total chromosomes undergo the mutation process.

\section{Parameter adaptation}

We consider the various adjustable parameters (APs) of cognitive radio which are denoted by a set $p=\left[p_{1}\right.$, $p_{2}, p_{3} \cdots p_{r}$ ] where $r$ denotes the number of adjustable parameters. These APs include FB, MOD, BER DR, PWR, ITPU and TOI. In order to select the optimal channel for communication, the CR should balance the multiple objectives with contradictory requirements to meet CR QoS need and cater the dynamic changes occur in the condition of channel along with the handling of the PU activity. the PU. The objective functions are denoted by $f=\left[f_{1}, f_{2}, f_{3} \cdots f_{q}\right]$, where $q$ represents the number of objective functions. For current case, the objective functions are DR, BER, PER, ITPU and TOI. To simplify the problem of PA we convert the multi objective optimization with various contradictory objective functions into a single objective function by using the relationship given in the [23] and normalizing the value of the objective function to the range 0 to 1 as follows:

$$
\begin{aligned}
f= & \sum_{i=1}^{n} w_{i} \cdot f_{i} \\
= & w_{d r} \cdot f_{d r+} w_{\text {pow }} \cdot f_{\text {pow }+} w_{\text {ber }} \cdot f_{\text {ber }}+w_{\text {itpu }} \cdot f_{\text {itpu }}+w_{T O I} \cdot f_{T O I} \\
& w=w_{1}+w_{2}+w_{3} \cdots+w_{m} \\
& w_{i} \geq 0 \quad \text { for } i=1,2 \ldots m
\end{aligned}
$$

where, $w=\left[w_{1}, w_{2}, w_{3} \cdots w_{m}\right]$ is a weight vector. We consider the four transmission modes depending upon the weight given to each objective function.

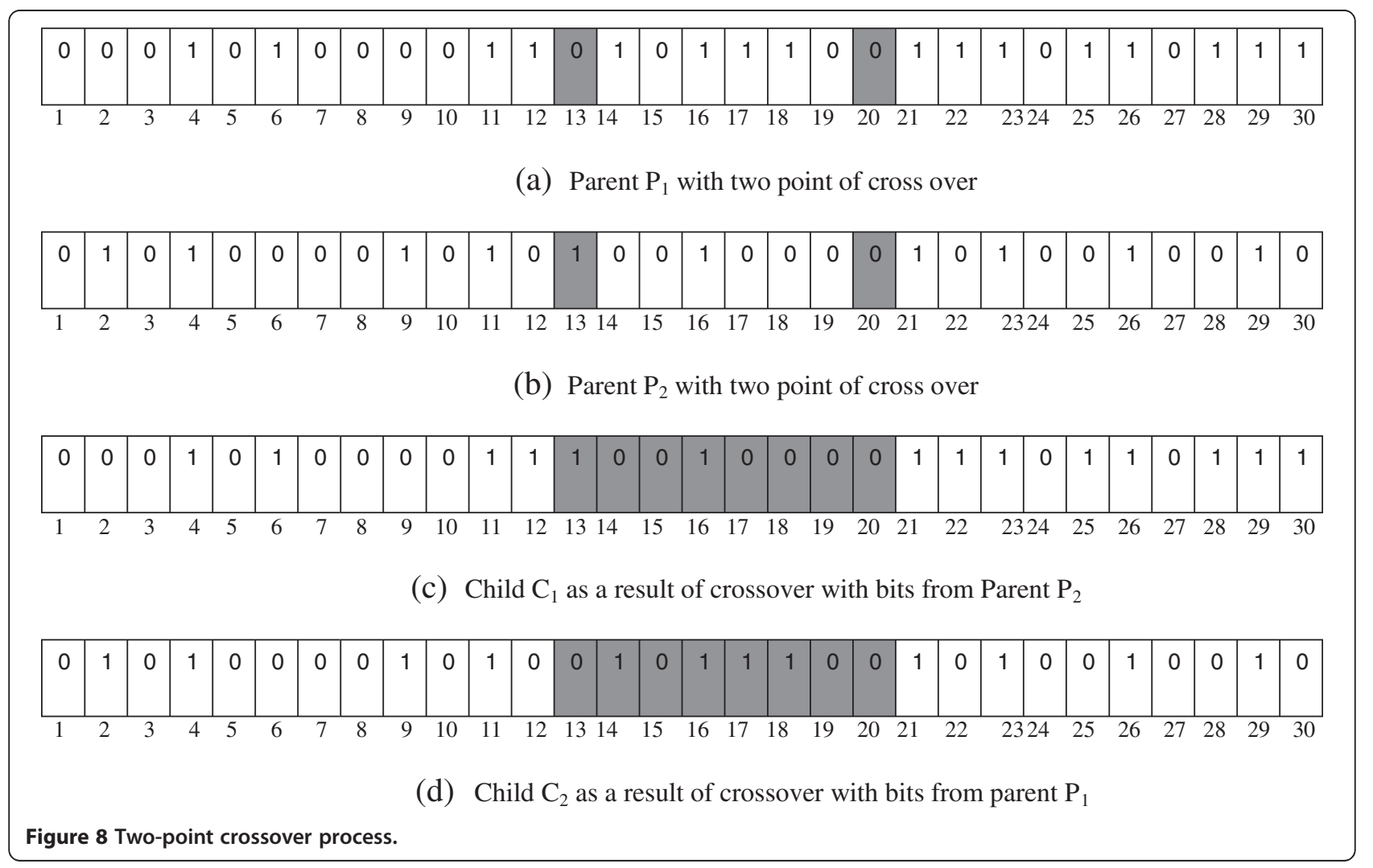




\section{Formulation of objective functions}

This sub section describes the mathematical modeling for the objective functions that we have considered in CSPA scheme. The

$$
\begin{aligned}
& f_{D R}= \begin{cases}\frac{d_{r}-d_{c}}{d_{r}} \text { for } d_{r}>d_{c} \\
1 \quad \text { otherwise }\end{cases} \\
& f_{\text {PWR }}=1-\frac{P}{P_{\max }} \\
& f_{\text {ber }}=\left\{\begin{array}{cc}
\frac{\log _{10}\left(\bar{P}_{b e}\right)}{\log _{10}\left(\bar{P}_{\text {min_ber }}\right)} \text { for } & P_{b e}<10^{-3} \\
0 & \text { otherwise }
\end{array}\right. \\
& f_{\text {ITPU }}=1-\frac{I}{I_{\max }} \\
& f_{\text {TOI }}=\frac{\eta}{\eta_{\max }}
\end{aligned}
$$

In equation (14), the $d_{r}$ represents the desired data rate and $d_{c}$ indicates the data rate available on channel [15]. The power optimization function is depicted in the equation (15) where $P$ indicates the power required for the transmission on the given channel and $P_{\max }$ is the maximum power available to the CR user. The $\bar{P}_{b e}$ in equation (16) represents the average BER of all the channels and $\bar{P}_{\text {minber }}$ is the minimum value of the BER among the available channels [15]. The factor $I$ shows the ITPU on the given channel while $I_{\max }$ is the maximum interference limit to the PU. Similarly $\eta$ is the future opportunity for the $\mathrm{CR}$ on the given channel while $\eta_{\max }$ represents the maximum value of the opportunity index. Depending upon the weightage given to each objective function we define the four transmission modes as shown in the Additional file 2: Table S2.

\section{Experimental results}

This section describes experimental results along with the detailed explanation of different simulation parameters. Moreover, it explains the significance of our simulation result in terms of channel switching, service time and power consumption.

\section{Experimental setup}

We consider the CRAHNs where each CR has capability of accurate sensing and history management. Although the given results are true for more generic cases yet we show our outcomes for specific values of the GA parameters. Additional file 3: Table S3 specifies the basic GA parameters that we have considered for our implementation.
The value of the crossover rate is 0.5 and it indicates that the six chromosomes are selected to form the next generation. The mutation rate is selected based upon the existing work in the channel selection process. The 200 iterations are performed to get the desired solution (chromosome).

\section{The CR user requirements}

The CSPA selects the channel according to the requirement of the CR users, the PU activity and the QoS parameters of CR. For the current case the desired QoS parameters of the $\mathrm{CR}$ are given in Additional file 4: Table S4. The CR user needs 265 kbps with maximum tolerable BER of $10^{-5}$ to follow its transmission.

\section{Simulation results}

To find out how the objective function gets improved with the number of iterations, the simulation results are repeated 100 times with the 200 GA's iterations and the best chromosome is selected. The sample solution of CSPA scheme considers the parameters given in Additional file 4: Table S4 and its values are shown in Figure 9. The given solution ensures the required QoS parameters for the $C R$ users with the $F B=400 \mathrm{MHz}$, $\mathrm{MOD}=\mathrm{QPSK}, \quad \mathrm{BER}=10^{-9}, \quad \mathrm{PWR}=3 \mathrm{dBm}, \quad \mathrm{ITPU}=0.25$, TOI $=0.9394$. The TOI achieves its optimal value and hence provides the required level of DR and other objectives mention in the Additional file 4: Table S4.

We also compute and plot the average objective function values for four different operation modes given in the Additional file 2: Table S2 to test the effectiveness of CSPA scheme. The 200 iterations are performed to find out the best solution. Each mode of operation focuses on different objectives and is appropriate for different application and situation. The MT mode in Figure 10 (a) maximizes the average throughput of the CR user. It is more appropriate for those application demanding higher data rates such as broad band video. The PS mode is effective for power sensitive application and suitable for the CR sensor networks and text messaging. The RB mode indicates the reliability mode and it helps to reduce the BER for different applications like emergency applications. The BQoS mode is the core development of this article and it helps to reduce number of interruptions/channel switchings with PUs. Moreover, it also helps to quickly serve the CR user and to save the extra power compared with PS mode by avoiding the re transmission owing to the collision occurs with the PU. The BQoS mode is suitable for CRAHNs and the cognitive radio sensor networks.

Figure 11 describes the different modes of operation and the value of respective genes. For simplicity, we have shown the values of different genes for the first 50 iterations. The MT mode shown in Figure 11 (a) describes 


\begin{tabular}{|l|l|l|l|l|l|l|l|l|l|l|l|l|l|l|l|l|l|l|l|l|l|l|l|l|l|l|l|l|l|}
\hline 0 & 1 & 1 & 1 & 0 & 1 & 0 & 0 & 1 & 1 & 0 & 0 & 0 & 1 & 1 & 1 & 0 & 1 & 0 & 0 & 0 & 1 & 0 & 0 & 1 & 1 & 1 & 1 & 1 & 0 \\
& & & & & \\
\hline
\end{tabular}

Figure 9 Sample GA provided solution using CSPA.

the variations of different genes across different iteration. The data rate objective function has the $60 \%$ weight hence the data rate starts maturing and maximizing its value after a few iterations and reach close to the optimal value of $1.620 \mathrm{kbps}$ after only 30 iterations. Although the maximum value of DR is $1.65 \mathrm{kbps}$ but for simplicity, we assume the mid value for each level of DR. Based on this assumption the maximum possible value of DR is $1.62 \mathrm{kbps}$. However, the values of other genes are not ideal but optimal owing to the less weight to their objective functions. Similarly in PS mode, the more percentage weight is given to the objective function of minimizing power and the required power reduces to few miliwatts (mw) very quickly in a few iterations. For simplicity we plot the power in mw. The BQoS mode in part (d) of Figure 11 shows the outcomes for our new added TOI gene. In this case $60 \%$ weight is given to the TOI gene which achieves its maximum value after 25 iterations and the rest of parameters are also approaching to their respective optimal values. Hence, CSPA converges to the desired solution very quickly.

The comparison on the basis of the channel switching and average service time is shown in Figure 12. The CSPA shows significantly large improvement as compared to the random channel selection schemes. We show the comparison for six different file sizes of CR user.

In Figure 12 (a), the comparison is shown on the basis of channel switchings. To transmit a data file of size $2 \mathrm{~Kb}$, by using the CSPA the CR needs to perform channel switching only 7 times while the random channel selection scheme requires 39 switchings which is almost 5.5 times higher as compared to CSPA scheme. The performance of CSPA is even much better for video application that needs to transmit large amount of data (a)

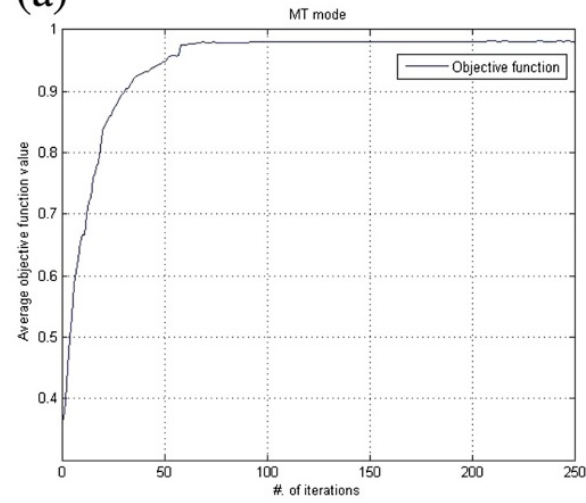

(b)

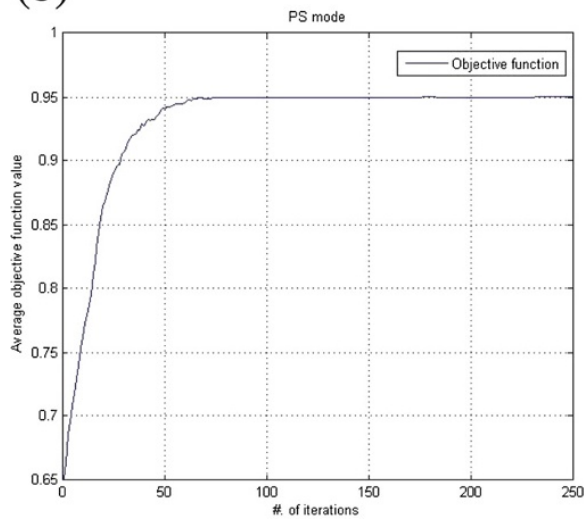

(c)

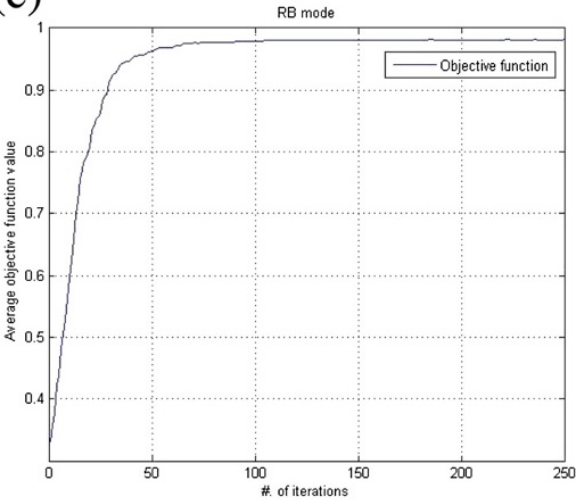

(d)

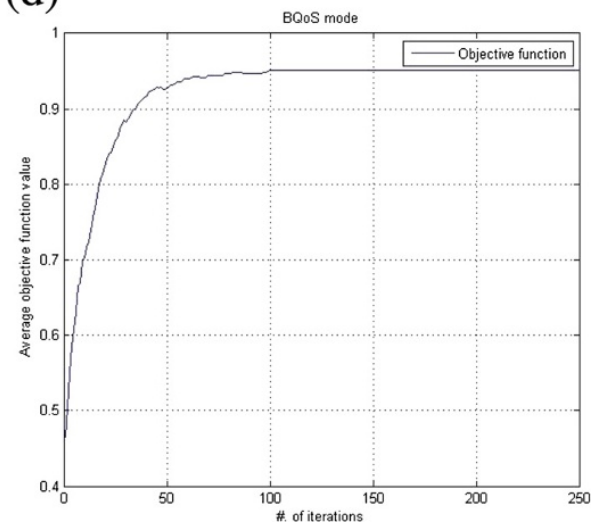

Figure 10 The objective function for different modes of operation. 

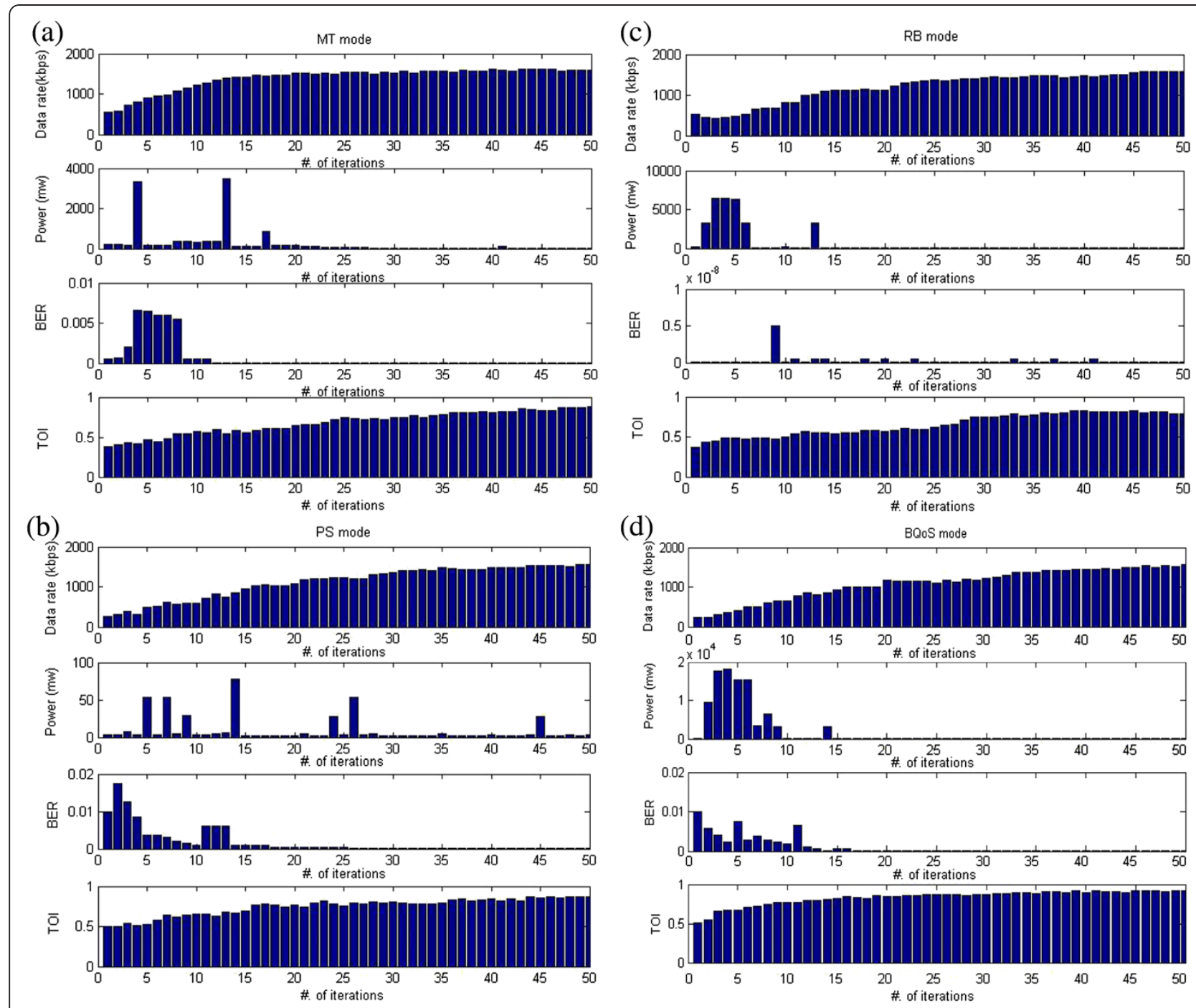

(d)

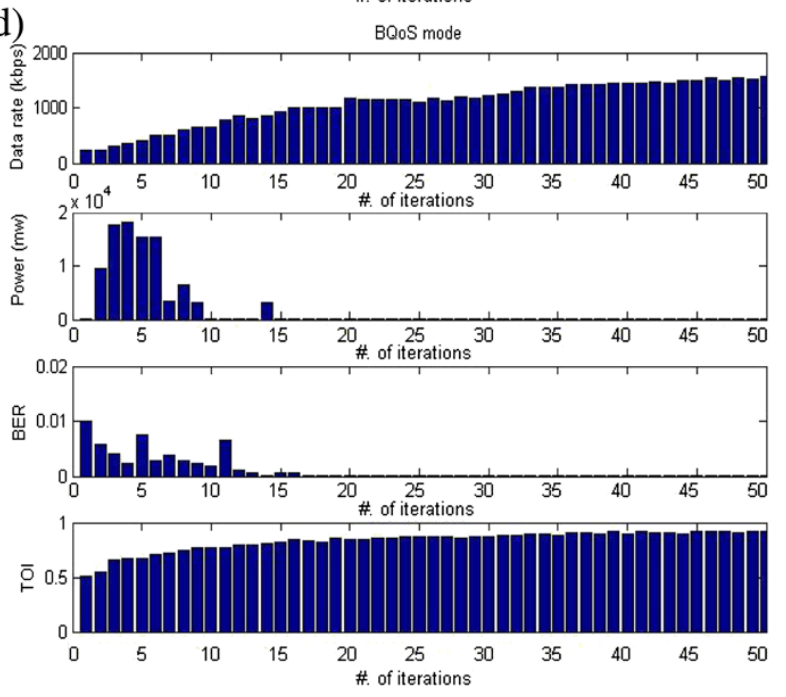

Figure 11 Sample result for different modes of operation.

as CSPA shows better performance for large data files. Hence, the performance of the CSPA scheme is much better than the traditional random channel selection schemes in terms of the channel switchings.

In Figure 12 (b), the comparison is shown on the basis of the time taken to transmit a data of the CR user. For the transmission of $2 \mathrm{~Kb}$ data file, the $\mathrm{CR}$ takes only 84.5 milliseconds ( $\mathrm{ms}$ ) while the random channel selection scheme requires $149.5 \mathrm{~ms}$ which is twice higher as compared to CSPA scheme. Moreover, the performance gain of the CSPA is even more significant for large data files. Although the GA consumes time in the selection of the best channel however GA selection time is considerably less as compared to the saving time.

Figure 13 depicts the outcomes and benefits of our new added genes in terms of number of channel switchings that the CR needs to perform on the arrival of the PU. It is clear from the line graph in Figure 13 that the number of channel switchings decrease exponentially with the increase in the percentage weight of the TOI gene. By only giving the $10 \%$ weight to the TOI gene, the switchings are reduced by $54.2 \%$. The channel switchings are reduced by $76.25 \%$ at the $40 \%$ TOI gene weight. Therefore, the given scheme is more robust against the issue of interruptions or collisions with the PUs.

In Figure 14 we have shown the effectiveness of our scheme in saving the transmission power using the $B$ QoS mode with weight vector $\left[\mathrm{w}_{5}=60 \%, \mathrm{w}_{1}=\mathrm{w}_{2}=\mathrm{w}_{3}=\right.$ $\left.\mathrm{w}_{4}=10 \%\right]$. The CSPA reduces number of channel switchings which helps to save the transmission power. For simplicity we use the average power factor for indicating the amount of saved power. The average power factor represents the power required to transmit the data in a given time slot and it is indicated by $\mu$. The equation (19) describes the average power saving factor $P_{\text {sav }}$ achieved 


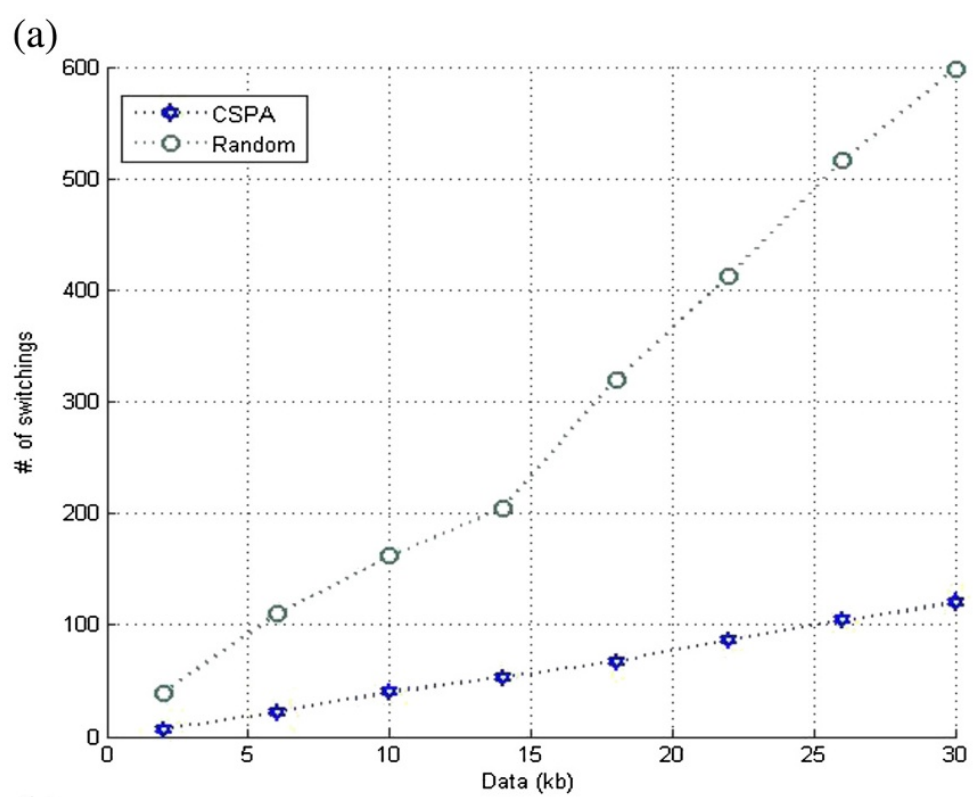

(b)

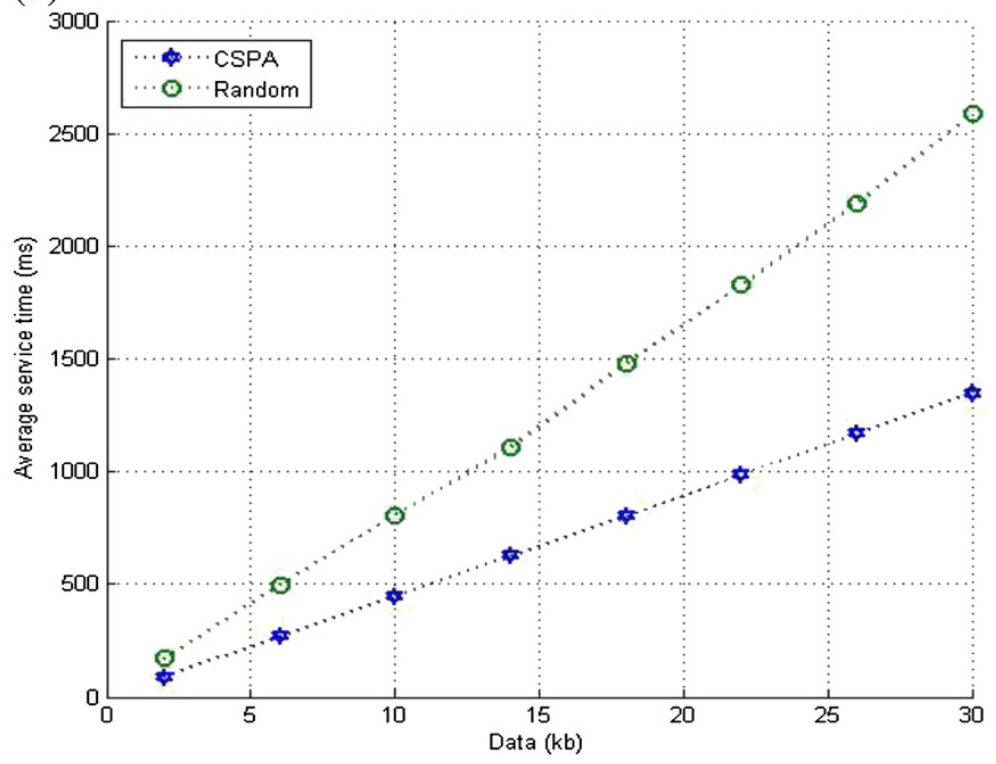

Figure 12 Comparison (a) channel switchings (b) average service time.

through CSPA scheme. The $S_{R}$ represents the switchings for random channel selection scheme and $S_{\text {cspa }}$ indicates the switchings occur during the CSPA scheme. The factor $\mu$ and $\beta$ quantifies the average power per bit and data for a given time slot respectively. We have shown the factor $P_{\text {sav }}$ for three different values of $\mu$ and $\beta$ to describe the power saving capability of CSPA scheme.

$$
P_{\text {sav }}=\left(S_{R}-S_{\text {cspa }}\right) * \mu * \beta
$$

The given simulation results makes CSPA suitable for power sensitive applications like cognitive radio sensor networks.

\section{Conclusion}

The channel selection and parameter adaptation are the most critical functions for CR user in CRAHNs. An optimal channel selection scheme would help to provide the better QoS to the CR users and operate inconsistence with the PU without creating any unpleasant effects on the ongoing communication of the PU. The both communication parties can perform their tasks without interference to each other. On the other hand the selection of channel on random basis without considering the PU activity would result in the higher number of channel switchings which cause the delay in the service time and wastage of the precious power. From 


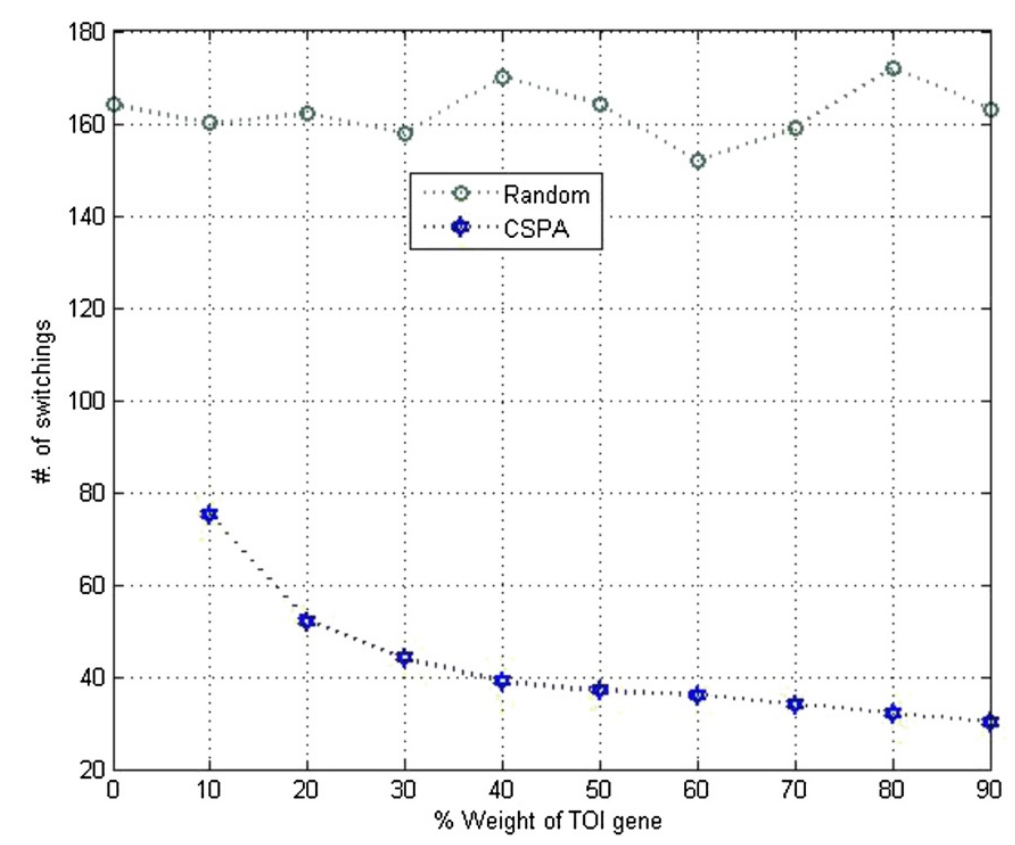

Figure 13 Comparison based on the channel switchings by varying weight of TOI gene.

the PU perspective, it is also desirable that the CR causes lease collisions and operates under tolerable interference range.

In this article, we proposed a channel selection and parameter adaptation scheme and formulate the problem for CRAHNs using the GA by considering all the important factors including the channel characteristics, the PU arrival activity and the QoS requirement of the CR users.

We also propose a method to model the activity of the $\mathrm{PU}$ and also formulate the mathematical equations to compute the transmission opportunity index which

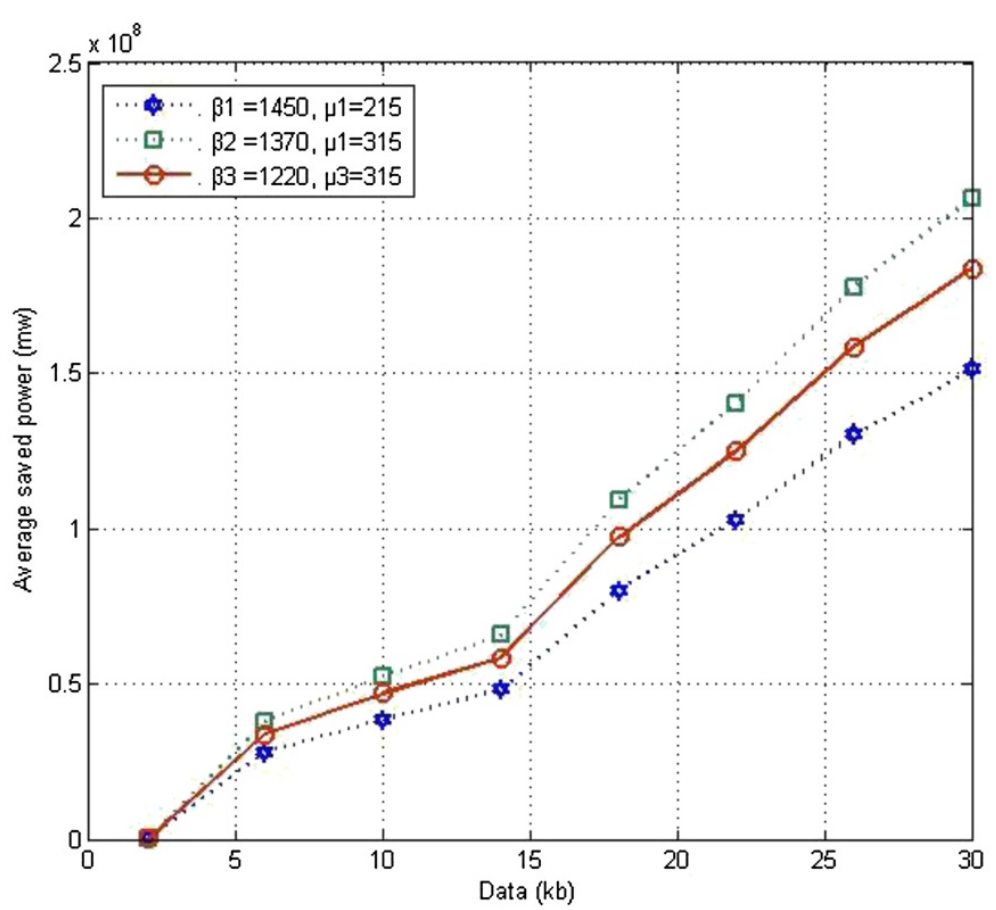

Figure 14 The saved power using CSPA. 
indicates the duration for the future availability of channel to the CR without interruptions from the PU. We add this information in the chromosome to incorporate the effect of the PU activity inside GA based decision making process for selecting the optimal channel. The solution is evolved through GA which performs the genetic operation and then $\mathrm{CR}$ user adapts their parameters according to the selected channel. We also define four transmission modes depending upon the weight given to the respective objective function. The four modes are defined and their effectiveness is discussed according to their applications in the real implementation.

Simulation results show that including the PU activity within the gene structure helps to significantly reduce the number of channel switchings, minimizes the delay in the service time and saves the transmission power. The CSPA scheme shows a significant improvement in saving the transmission power that makes it suitable for power sensitive application such as cognitive radio sensor network.

In future, we are planning to develop a similar model using particle swarm optimization and show the comparison with the genetic algorithm in terms of their convergence time. Moreover, we are also developing a model to incorporate the channel usage patterns of other CR users in the CRAHNs.

\section{Additional files}

Additional file 1: Table S1. Gene structure.

Additional file 2: Table S2. Modes of operation.

Additional file 3: Table S3. The GA parameters.

Additional file 4: Table S4. The QoS parameters of the CR.

Competing interests

The authors declare that they have no competing interests.

\begin{abstract}
Acknowledgment
This research was supported by the MKE (The Ministry of Knowledge Economy), Korea, under the Convergence-ITRC (Convergence Information Technology Research Center) support program (NIPA-2012-C6150-1101-0003) supervised by the NIPA (National IT Industry Promotion Agency) and the Basic Science Research Program through the National Research Foundation of Korea (NRF) funded by the Ministry of Education, Science and Technology (2012009449).
\end{abstract}

Received: 3 April 2012 Accepted: 23 September 2012

Published: 21 November 2012

\section{References}

1. FCC, Second Report and Order And Memorandum Opinion and Order, ET Docket No. 08-260 (2008)

2. IF Akyildiz, W Lee, MC Vuran, S Mohanty, NeXt generation/dynamic spectrum access/cognitive radio wireless networks: a survey. J. Comput. Netw 50(13), 2127-2159 (2006)

3. IF Akyildiz, WY Lee, K Chowdhury, CRAHNs: cognitive radio ad hoc networks. J. Ad Hoc Netw. 7(5), 810-836 (2009)

4. N Nie, C Comaniciu, Adaptive channel allocation spectrum etiquette for cognitive radio networks. in IEEE DySPAN, 269-278 (2005)
5. $H$ Zheng, $L$ Cao, Device-centric spectrum management. in IEEE DySPAN 56-65 (2005)

6. A Saleem, L Kyung-Geun, Fair, efficient and power optimized spectrum sharing scheme for cognitive radio network. EURASIP J. Wirel. Commun. Netw (2011). doi:10.1186/1687-1499-2011-201

7. L Yang, L Cao, $\mathrm{H}$ Zheng, Proactive channel access in dynamic spectrum networks. J. Phy Commu 1(2), 103-111 (2008)

8. H Marko, P Sofie, M Aarne, Performance improvement with predictive channel selection for cognitive radios. in IEEE CogART Workshop, 1-5 (2008)

9. S Ki Won, K Seong-Lyun, Z Jens, Temporal spectrum sharing based on primary user activity prediction. IEEE Trans. Wirel. Commun. 9(12), 38483855 (2010)

10. T Vamsi Krishna, W Ping, N Dusit, Channel status prediction for cognitive radio networks. J. Wirel. Commun. Mob. Comput. (WCMC) 10, 1-13 (2010)

11. T Christian James Rieser, Biologically inspired cognitive radio engine model utilizing distributed genetic algorithms for secure and robust wireless communications and networking (Dissertation, Blacksburg, Virginia, 2004)

12. W Rondeaut, B Le, D Maldonado, D Scaperoth, C Bostian, Cognitive radio formulation and implementation. in IEEE CrownCom, 1-10 (2006)

13. TR Newman, BA Barker, AM Wyglinski, A Arvin, Cognitive radio implementation for wireless multicarrier transceivers. Wiley Wirel. Commun. Mob. Comput. 6, 1-14 (2006)

14. H Qin, J Su, Y Du, Multiobjective evolutionary optimization algorithm for cognitive radio networks. in IEEE IEEC'09, 164-168 (2009)

15. K Jae Moung, S Sung Hwan, H Ning, Z Guanbo, K Young Min, L Joo Kwan, Cognitive radio software testbed using dual optimization in genetic algorithm. in IEEE CrowCom, 1-6 (2008)

16. S Chantaraskul, K Moessner, Implementation of a genetic algorithm-based decision making framework for oppertunistic radio. IET Commun 4, 495-506 (2010)

17. C Si, TR Newman, EAM Wyglinski, Genetic algorithm-based optimization for cognitive radio networks. in Proc. 2010 IEEE Sarnoff Symposium, 1-6 (2010)

18. T Siddique, A Azam, Spectrum Optimization in Cognitive Radio Networks Using Genetic Algorithm (Blekinge Institute of Technology, Sweden, 2010)

19. TR Newman, R Rakesh, AM Wyglinski, JB Evans, GJ Minden, Population adaptation for genetic algorithm-based cognitive radios. in IEEE CrownCom, 279-284 (2007)

20. W Gardner, W Brown, Spectral correlation of modulated signal. Part Ianalog modulation. IEEE Trans. Commun COM-35, 584-594 (1987)

21. WA Gardner, W Brown, CK Chen, Spectral correlation of modulated signal. Part II_digital modulation. IEEE Trans. Commun. COM-35, 595-601 (1987)

22. I Hong, SH Sohn, JK Lee, JM Kim, DFS algorithm with ranking based on genetic algorithm in UHF portable system. in IEEE ISCIT, 454-459 (2009)

23. C You, H Kwon, J Heo, Cooperative TV spectrum sensing in cognitive radio for Wi-Fi networks. IEEE Trans. Consum. Electron. 57, 62-67 (2011)

doi:10.1186/1687-1499-2012-349

Cite this article as: Aslam and Lee: CSPA: Channel Selection and Parameter Adaptation scheme based on genetic algorithm for cognitive radio Ad Hoc networks. EURASIP Journal on Wireless Communications and Networking 2012 2012:349.

\section{Submit your manuscript to a SpringerOpen ${ }^{\circ}$ journal and benefit from:}

- Convenient online submission

- Rigorous peer review

- Immediate publication on acceptance

- Open access: articles freely available online

- High visibility within the field

- Retaining the copyright to your article

Submit your next manuscript at $>$ springeropen.com 